\title{
Retrobulbar steatitis and meningitis/empyema secondary to right otitis media, right otitis interna and an inflammatory polyp in a cat
}

\author{
Elena Fenollosa-Romero*, Olivier Taeymans, Pieter Nelissen, Giunio B. Cherubini and Claudia Busse \\ Dick White Referrals, Station Farm, London Road, Six Mile Bottom, Cambridgeshire CB8 OUH, UK
}

\begin{abstract}
The purpose was to describe a case of retrobulbar steatitis and meningitis in a cat caused by otitis media, otitis interna and an inflammatory polyp in the middle ear. Investigations included ophthalmic and neurological examinations, haematology and biochemistry, magnetic resonance imaging (MRI), cerebrospinal fluid (CSF) analysis and aerobic, anaerobic bacterial and fungal culture of material from the middle ear. A 6-year-old female neutered domestic-shorthaired cat presented with right-sided exophthalmos and resistance to retropulsion. Anisocoria, reduced corneal sensation and oculocephalic movements and low Schirmer tear test (STT1) were found. An MRI revealed the right external ear canal and tympanic bulla to be fluid filled with presence of a thickened contrast-enhancing mucosa. Contrast enhancement extended into the calvarium, as marked thickening and contrast enhancement of the pachymeninges, and further rostrally through the right orbital fissure into the orbit. The retrobulbar tissues were swollen, resulting in mild exophthalmos. Enrichment culture of material from the middle ear revealed Klebsiella pneumonia susceptible to marbofloxacin. The cat underwent a total ear canal ablation with removal of the cartilaginous cuff at the external auditory meatus and local debridement and curettage. A polypoid structure was removed from the middle ear. No bulla osteotomy was performed. Horner's syndrome was present immediately following surgery. The cat received a course of systemic dexamethasone $(1 \mathrm{mg} / \mathrm{cat} \mathrm{SID} / 3$ weeks) tapered off and marbofloxacin $(2 \mathrm{mg} / \mathrm{kg}$ SID/2 weeks). Follow up at 120 days showed no recurrence of the symptoms. The Horner's syndrome and low STT1 had resolved and returned to normal values respectively. This case highlights the importance of MRI in the investigation of retrobulbar diseases. MRI is a useful tool for the evaluation of the extent of the lesion and the appearance of adjacent structures in which additional potentially life-threatening abnormalities, such as meningitis, can be seen.
\end{abstract}

Keywords: Exophthalmia, Meningitis, Otitis media/interna, Polyp, Retrobulbar disease.

\section{Introduction}

Otitis in cats is usually a multifactorial problem (Kennis, 2013). The perpetuating factors, bacteria and yeast organisms that are the source of frustration in many canine otitis cases, are less frequently a problem in cats (Kennis, 2013). Intracranial infections originating from ear disease have been described in dogs and cats (Sorjonen, 1992; Sturges et al., 2006) but reports are sparse. In human medicine, the widely accepted use of antibiotics to treat infectious otitis media has dramatically decreased the number of complications however, they still occur (Penido et al., 2005). Intracranial extension of ear disease represents the most life-threatening condition of otitis media in people, requiring immediate and precise therapeutic intervention (Penido et al., 2005).

A recent publication reviewed the treatment and diagnosis of otogenic intracranial infection in dogs and cats (Sturgess et al., 2006). A case of retrobulbar abscess and suspected intracranial extension in a cat has been described in the literature (Barrs et al., 2007) but no reports about concomitant retrobulbar, meningeal and otogenic involvement have been published before. This case report presents an unusual case of meningitis and retrobulbar disease as a complication of chronic otitits media and interna.

\section{Case Details}

A six-year-old female neutered domestic short-haired cat presented with an eight-week history of right-sided otitis externa that had been treated with different topical ear treatments including miconazole nitrate, prednisolone acetate and polymixin B sulphate (Surolan ${ }^{\circledR}$, Jansen Pharmaceutica, Belgium) BID/10 days and systemic non steroidal anti-inflammatory drugs (meloxicam $0.05 \mathrm{mg} / \mathrm{kg}$ po SID $/ 14$ days; Metacam ${ }^{\circledR}$ (Boehringer, Ingelheim, Vetmedica, Germany)). Recent exophthalmia of the right eye had been noticed. The cat was anorexic and lethargic. A full ophthalmic examination performed by a board-certified veterinary ophthalmologist revealed exophthalmos of the right eye and resistance to retropulsion, pain on fully opening the mouth, anisocoria with a mildly dilated pupil in the right eye in normal lighting conditions, reduced corneal sensation and reduced 
oculocephalic movements and a paraxial superficial corneal ulcer (Fig. 1).

The eye was visual and had a reduced direct pupillary light reflex and a normal consensual pupillary light reflex (right to left eye).

Examination of the left eye was considered normal. Schirmer tear test (STT1) values (Intervet Inc., Merck Animal Health, USA) were $8 \mathrm{~mm} / \mathrm{min}$ in the right eye and $17 \mathrm{~mm} / \mathrm{min}$ in the left and intraocular pressures were 18 and $20 \mathrm{~mm} \mathrm{Hg}$ in the right and left eye respectively (Tonovet ${ }^{\circledR}$ Icare, Finland). Based on the ophthalmic examination, a retrobulbar problem was suspected and further investigations were recommended.

A neurological examination by a board certified neurologist revealed no further neurological deficits. A full biochemistry and hematology profile revealed the following: mild hyperproteinemia $(80 * ; 54-78)$ and hyperglobulinemia $\left(49^{*} ; 20-45\right)$, low urea $\left(2.3^{*} ; 6-10\right)$, lipase elevation $(131 * ; 8-26)$, leukocytosis (21.48*; 5.5-19.5) and neutrophilia (19.63*; 2.5-12.5). The patient was sedated with methadone $(0.3 \mathrm{mg} / \mathrm{kg} \mathrm{IV}$; Comfortam ${ }^{\circledR}$, Dechra, Bladel, The Netherlands) and dexmedetomidine (2 mcg/kg IV; Dexdomitor ${ }^{\circledR}$, Vetoquinol, Buckinghamshire, UK), induced with alfaxolone (Alfaxan®, Jurox, Worcestershire, UK) to effect $(4 \mathrm{mg} / \mathrm{cat})$, intubated and general anaesthesia maintained with isofluorane (Isothesia ${ }^{\circledR}$, Henry Schein, Berkshire, UK) in oxygen.

Magnetic resonance imaging (MRI), including the following sequences: short TI inversion recovery in dorsal plane (DORS STIR) / post-contrast spin-echo T1 in a sagittal plane (SAG SE T1+c) / fast-spin-echo T2, spin-echo T1, post-contrast spin-echo T1 in transverse planes (TRANS FSE T2, SE T1, SE T1+c), as well as a post-contrast T1-weighted isotropic 3D gradientrecalled echo volumetric sequence (3D GRE T1+c) were performed.

The right external ear canal and tympanic bulla were fluid filled and showed a thickened, contrast-enhancing mucosa. This contrast enhancement extended into the calvarium through the inner ear, resulting in marked thickening and contrast enhancement of the pachymeninges around the right side of the cerebrum. Further extension was then seen through the right orbital fissure, resulting in retrobulbar steatitis. The latter resulted in mild exophthalmos. Contrast enhancement was also extending through the right alar/oval foramen and tracking around the trigeminal nerve.

The right medial retropharyngeal lymph node was moderately enlarged. The left tympanic bulla was fluid filled, without similar contrast-enhancement as on the contralateral side (Fig.2 and 3). Cerebrospinal fluid (CSF) was collected from the cisterna magna.

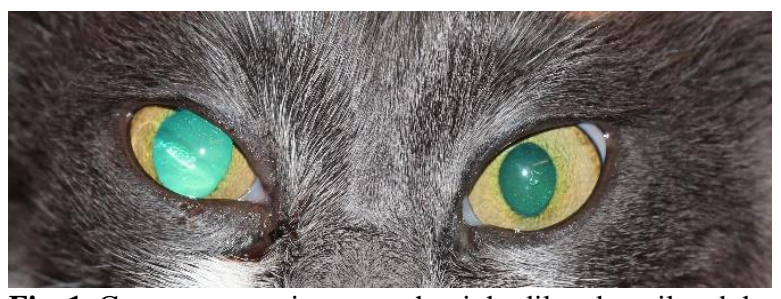

Fig. 1. Cat at presentation: note the right dilated pupil and the paraxial corneal ulcer.

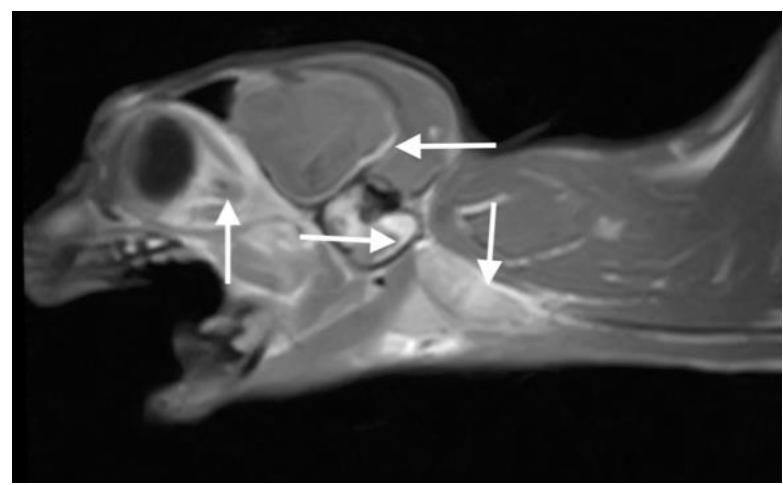

Fig. 2. Parasagittal SE T1+c image. Note arrows indicate: retrobulbar steatitis $(\boldsymbol{\uparrow})$, meningitis $(\leftarrow)$, otitis media $(\rightarrow)$, medial retropharyngeal lymphadenitis $(\downarrow)$.

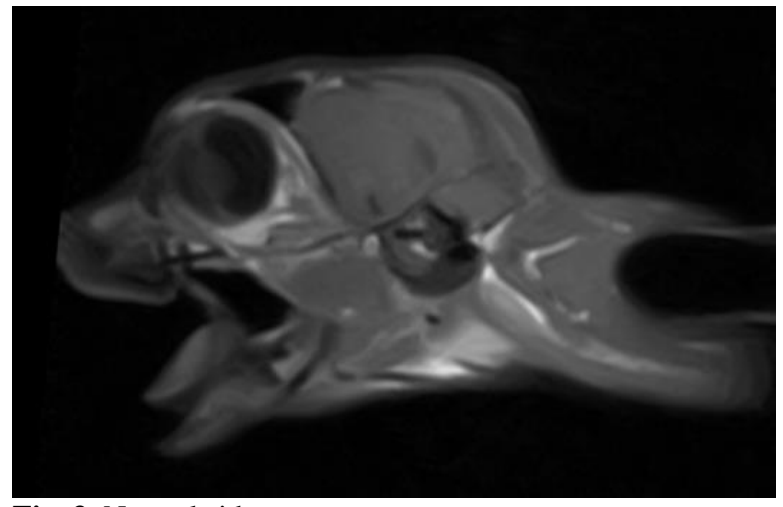

Fig. 3. Normal side.

A course of broad-spectrum antibiotic with clindamycin (Antirobe ${ }^{\circledR}, \quad$ Zoetis, UK Limited; $8.7 \mathrm{mg} / \mathrm{kg} / \mathrm{BID} / 1$ week) and amoxicillin/clavulanate (Noroclav®, Norbrook Lab GB Ltd; $18 \mathrm{mg} / \mathrm{kg} / \mathrm{BID} / 1$ week) was started in association with antiinflammatory dexamethasone (Auden Mckenzie, UK; $1 \mathrm{mg} /$ daily/on tapering dosage 3 weeks).

Resistance to retropulsion improved 24 hours after starting with systemic corticosteroids and antibiotics. The reduced corneal sensation remained unchanged. Mirtazaprine (Medreich PLC, Feltham, UK; $2 \mathrm{mg} /$ cat/every other day) was started as an appetite stimulant as the cat was not eating. Topical lubrication (Lubrithal ${ }^{\circledR}$, Dechra Vet Products, Uldum) was started in the right eye every 4 hours/ 3 days. 
Forty-eight hours after initial presentation the anisocoria had improved and the pupillary light reflex was more complete. The epithelial defect was reepithelizing.

Three days after presentation, a board-certified surgeon assessed the patient to plan a total ear canal ablation. The same pre-medication and anaesthetic protocol described above was followed. A $0.5 \mathrm{mg} / \mathrm{kg} / \mathrm{IV}$ bolus of ketamine (Ketavet $100 \mathrm{mg} / \mathrm{ml}$, Zoetis, Ireland Limited) followed by infusion at $(0.5 \mathrm{mg} / \mathrm{kg} / \mathrm{hr})$ was given at the beginning of the surgical procedure. External ear canal ablation was performed in a standard manner. The facial nerve was identified and was preserved, along with all the surrounding larger vessels. The ear canal was transected at the level of the external auditory meatus (EAM).

The cartilage cuff at the auditory meatus was resected using small rongeurs and sharp dissection. Inflammatory tissue surrounding the horizontal part was debrided and submitted for aerobic and anaerobic bacterial and fungal culture. The external auditory meatus was filled with debris and the tympanic membrane was ruptured. The middle ear was filled with polypoid tissue that was removed in its entirety using Alligator forceps. Gentle curettage of the tympanic chamber was performed through the EAM, which was then flushed with saline. Gentle debridement and curettage of the middle ear chamber was continued until no inflammatory tissue or epithelial tissue was present. The deeper and subcutaneous tissues were reconstructed with interrupted sutures and a simple continuous suture using 4-0 poliglecaprone 25 (Monocryl®, Ethicon). The skin was sutured with an intradermal 4-0 poliglecaprone 25 (Monocryl®, Ethicon) continuous suture pattern. The polypoid structure was submitted for histopathology.

Horner's syndrome (ptosis of the upper lid, third eyelid protrusion, miosis and enophthalmia) was evident on the right side immediately following surgery. The reduced corneal sensation remained present. Topical fusidic acid (Isathal®, Dechra, Uldum) BID/14 days was started after the surgical procedure. The patient was discharged 72 hours after surgery. The epithelial defect was still present.

CSF analysis revealed blood contamination. Histology results of the mass removed were consistent with an inflammatory polyp. Enrichment culture results of the right ear canal yielded a growth of Klebsiella Pneumoniae susceptible to marbofloxacin. Treatment was therefore changed to marbofloxacin (Marbocyl®, Vetoquinol, UK; $2 \mathrm{mg} / \mathrm{kg}$ once daily for 2 weeks). Reexamination three weeks after initial presentation revealed a bright and alert cat. The surgical site had healed without complications. Ophthalmic examination revealed reduction of the Horner's syndrome signs and a completely re-epithelized ulcer. An area of fibrosis was visible at the previously ulcerated area. The STT1 was $5 \mathrm{~mm} / \mathrm{min}$ in the right eye and $25 \mathrm{~mm} / \mathrm{min}$ in the left eye. Intraocular pressure readings were within normal limits. Sodium hyaluronate $0.15 \%$ (Hyabak®, Lab Thea, Barcelona) BID/120 days was started and continued until the last re-examination performed by the referring veterinarian 120 days after initial presentation. At this stage, the STT1 readings were 17 and $20 \mathrm{~mm} / \mathrm{min}$ in the right and left eye respectively. No further signs of the Horner's syndrome were present.

\section{Discussion}

The widespread use of antibiotics has dramatically decreased the incidence of intracranial complications of otitis and sinusitis in human medicine (Aimoni et al., 2005). The recognition of potential complications in the presence of otitis media/interna (OMI) is paramount to a successful outcome, because if left undiagnosed they can be life threatening. There are few published reports that describe the clinical presentation, diagnosis, or subsequent treatment and outcome of intracranial extension of OMI in dogs and cats, suggesting that the disorder is uncommon (Sturges et al., 2006). To the author's knowledge, there are no reports available in the literature where the clinical presentation involves the retrobulbar space. Ocular signs of retrobulbar disease were present in this case with resistance to retropulsion and pain opening the mouth. Third eyelid protrusion was absent in this case however third eyelid protrusion was only present in $28 \%$ of the cases in a retrospective study of retrobulbar neoplasia (AttaliSoussay et al., 2001).

The most likely anatomic pathway for entry of organisms from the inner ear into the brainstem of dogs and cats is along the nerves and vessels of the internal acoustic meatus (Sturges et al., 2006). Involvement of the retrobulbar space in this case is suspected to have extended through the right orbital fissure into the orbit as seen on the MRI images. In human medicine infection spreading from the ear could occur via osteothrombophlebitis of small vessels or through a preformed pathway, such as the labyrinth or the endolymphatic channels, or it could be related to developmental or traumatic bony defects, by bony erosion due to cholesteatoma, or granulation with underlying osteitis (Samuel et al., 1986).

Other presenting signs included anisocoria, which could likely be explained by involvement of the parasympathetic fibres to the pupil within the orbit through the orbital foramen. Low STT1 in the affected eye was also initially present likely due to involvement of the parasympathetic fibres to the lacrimal gland. There also was reduced corneal sensation that is explained by trigeminal nerve involvement, as on MRI contrast enhancement was visible extending through the right alar/oval foramen and tracking around the 
trigeminal nerve (Fig. 2). The cause for the corneal ulceration was likely a combination of the inability to achieve full closure of the eyelids caused by the exophthalmia and the decreased corneal sensation that would have decreased the stimulus on the efferent arm of the blinking reflex, consequently reducing the reflex tear production.

Clinical signs of orbital disease may be subtle and are often non-specific with regard to the aetiology (Dennis, 2000), the diagnosis and characterisation of orbital disease may therefore be challenging. Successful treatment relies on accurate diagnosis (Dennis, 2000). Although ultrasound of the retrobulbar space can be helpful in some cases and its affordability makes it available to almost all patients; acquisition of the images requires an experienced ultrasonographer. Even in experienced hands, inconclusive results are common and further imaging techniques, such MRI, are required. In this case, central nervous system (CNS) involvement was only diagnosed through the use of MRI; and an ultrasound was not performed. Ultrasonography would likely have missed it with potential life threatening consequences to the cat. In a recent study, MRI produced detailed images of orbital tissues and provided more information about the extent of pathology than other imaging techniques (Dennis, 2000). MRI is the imaging modality of choice when intracranial extension of OMI is suspected (Sturges $e t$ al., 2006).

To treat the middle ear disease, surgical drainage of the tympanic bulla and the cranial vault combined with appropriate antibiotics, followed by long-term, culturespecific antibiotics, are recommended (Sturges et al., 2006). The ventral approach to the bulla and the lateral approach combined with ablation of the vertical and horizontal parts of the external acoustic meatus were the common surgical approaches described in another study (Trevor and Martin, 1993). Although Staphylococcus spp., E coli, Pseudomonas aeruginosa, and Proteus mirabilis typically have been reported in association with otitis media/interna in veterinary literature; on the basis of the results in the group of animals in Sturges' study other organisms, such as Enterococcus spp., Streptococcus spp., anaerobic organisms in dogs and cats, and Pasteurella multocida in cats, should be considered as potential pathogens (Sturges et al., 2006). Klebsiella Pneumoniae that was cultured in this case was also reported in another retrospective study (Radaelli and Platt, 2002).

In people, neurologic sequelae after meningitis secondary to acute OMI are thought to develop as a result of the effects of inflammatory mediators and cytokines (Roos, 1995) Anisocoria and reduced corneal sensation were present in this case, which were not solely explained by the presence of a retrobulbar problem but through the CNS involvement. However these neurological deficits improved following surgical and medical treatment and no sequelae were left after 120 days.

An anti-inflammatory dose of steroids (dexamethasone $1 \mathrm{mg} / \mathrm{cat} /$ daily) combined with systemic broadspectrum antibiotics were initially started in this case. Systemic steroids were continued for 21 days. Glucocorticoids that are routinely administered to these patients to address the neurological involvement in human medicine have not been found to interfere with antibiotic treatment (Roos, 1995).

Although the number of animals in the Sturges' study was small, judicious use of glucocorticoids appears to have been beneficial, or not obviously detrimental (Sturges et al., 2006).

Complications following bulla osteotomy curettage such as Horner's syndrome are common and were present in this case. It resolved 90 days after surgery. In the Sturges' review immediate postoperative complications have been reported to be common but short-term and included Horner's syndrome (11 cats), facial nerve paralysis ( 5 cats), and otitis interna ( 2 cats) (Sturges et al., 2006). In this case, the Horner's syndrome and the initially low STT1 had disappeared and returned to normal values respectively by day 120 as reported by the referring veterinarian.

Although prognostic indicators that are based on the neurologic presentation may be poor, the long-term outcome for cats and dogs with intracranial extension of OMI appears to be excellent in most instances after aggressive surgical and medical therapy (Sturges $e t a l$., 2006). In this case involvement of the retrobulbar space did not appear to add further complications and a full recovery was achieved.

\section{Conclusion}

This case highlights the importance of MRI in the investigation of retrobulbar diseases. MRI is a useful tool for the evaluation of the extent of the lesion and the appearance of adjacent structures in which additional potentially life-threatening abnormalities, such as meningitis, can be seen.

\section{Conflict of interest}

The authors declare that there is no conflict of interests.

\section{References}

Aimoni, C., Pelucchi, S., Grasso, D.L., Libanore, M. and Martini, A. 2005. Bacterial meningitis complicating suppurative otitis media and sinusitis. Otolaryngol. Head Neck Surg. 132(6), 965-966.

Attali-Soussay, K., Jegou, J.P. and Clerc, B. 2001. Retrobulbar tumors in dogs and cats: 25 cases. 2001. Vet. Ophthalmol. 4(1), 19-27.

Barrs, V.R., Nicoll, R.G., Churcher, R.K., Beck, J.A. and Beatty, J.A. 2007. Intracranial empyema: Literature review and two novel cases in cats. J. Small Anim. Pract. 48(8), 449-454. 
Dennis, R. 2000. Use of magnetic resonance imaging for the investigation of orbital disease in small animals. J. Small Anim. Pract. 41(4), 145-155.

Kennis, R.A. 2013. Feline otitis: diagnosis and treatment. Vet. Clin. North Am. Small Anim. Pract. 43(1), 51-56.

Penido Nde, O., Borin, A., Iha, L.C.N., Suguri, V.M., Onishi, E., Fukuda, Y. and Cruz, O.L. 2005. Intracranial complications of otitis media: 15 years of experience in 33 patients. Otolaryngol. Head Neck Surg. 132, 37-42.

Radaelli, S.T. and Platt, S.R. 2002. Bacterial meningoencephalomyelitis in dogs: a retrospective study of 23 cases (1990-1999). J. Vet. Intern. Med. 16(2), 159-163.

Roos, K.L. 1995. The use of adjunctive therapy to alter the pathophysiology of bacterial meningitis. Clin.
Neuropharmacol. 18(2), 138-147.

Samuel, J., Fernandes, C.M. and Steinberg, J.L. 1986. Intracranial otogenic complications: a persisting problem. Laryngoscope 96(3), 272-278.

Sorjonen, D.C. 1992. Myelitis and meningitis. Vet. Clin. North Am. Small Anim. Pract. 22(4), 951-964.

Sturges, B.K., Dickinson, P.J., Kortz, G.D., Berry, W.L., Vernau, K.M., Wisner, E.R. and LeCouteur, R.A. 2006. Clinical signs, magnetic resonance imaging features, and outcome after surgical and medical treatment of otogenic intracranial infection in 11 cats and 4 dogs. J. Vet. Intern. Med. 20(3), 648-656.

Trevor, P.B. and Martin, R.A. 1993. Tympanic bulla osteotomy for treatment of middle-ear disease in cats: 19 cases (1984-1991). J. Am. Vet. Med. Assoc. 202(1), 123-128. 